\title{
Bile Flow Phantom Model and Animal Bile Duct Dilation Model for Evaluating Biliary Plastic Stents with Advanced Hydrophilic Coating
}

\author{
Chang-II Kwon ${ }^{1}$, Gwangil Kim², Seok Jeong ${ }^{3}$, Won Seop Lee ${ }^{3}$, Don Haeng Lee ${ }^{3}$, Kwang Hyun Ko ${ }^{1}$, Sung Pyo Hong ${ }^{1}$, and Ki \\ Baik Hahm ${ }^{1}$ \\ ${ }^{1}$ Digestive Disease Center, ${ }^{2}$ Department of Pathology, CHA Bundang Medical Center, CHA University, Seongnam, and ${ }^{3}$ Digestive Disease Center, \\ Department of Internal Medicine, Inha University School of Medicine, Incheon, Korea
}

See editorial on page 499.

Background/Aims: The efforts to improve biliary plastic stents (PSs) for decreasing biofilm formation and overcome short patency time have been continued. The aim of this study is to evaluate the effect of advanced hydrophilic coating for patency and biodurability of PS. Methods: Using an in vitro bile flow phantom model, we compared patency between prototype PS with hydrophilic coating (PS+HC) and prototype PS without hydrophilic coating (PS-HC). We performed an analysis of the degree of luminal narrowing by microscopic examination. Using an in vivo swine bile duct dilation model made by endoscopic papillary closure and stent insertion, we evaluated biodurability of hydrophilic coating. Results: In the phantom model, PS+HC showed less biofilm formation and luminal narrowing than PS-HC at 8 weeks $(p<0.05)$. A total of 31 stents were inserted into the dilated bile duct of seven swine models, and 24 stents were successfully retrieved 8 weeks later. There was no statistical difference of stent patency between the polyethylene PS+HC and the polyurethane PS+HC. The biodurability of hydrophilic coating was sustained up to 8 weeks, when assessing the coating layer by scanning electron microscopy examination. Conclusions: Advanced hydrophilic coating technology may extend the patency of PS compared to uncoated PS. (Gut Liver 2016;10:632-641)

Key Words: Plastic stents; Biliary stents; Hydrophilic coating; Biodurability

\section{INTRODUCTION}

Plastic stents (PSs) are easy to apply and less expensive than self-expandable metal stents (SEMS) in biliary obstructive diseases, in addition to being easier to remove. Limitations, however, can include insufficient luminal diameter and short patency period. PS are prone to occlusion by biliary sludge and/ or development of bacterial biofilm formation. ${ }^{1,2}$ According to a prospective study comparing different diameters of PS, there was no additive draining effect of PS larger than $10 \mathrm{~F}^{3}$ Also, stent material and its shape did not add advantages in draining effect. $^{4-6}$

Clogging of PS was mainly associated with biliary sludge and biofilm that can be synergistically affected by mixed bacterial infections and dietary fiber. ${ }^{7-9}$ As effort to overcome clogging of PS, innovative attempts or newly improved PS have been continued, focusing on improving biodurability (e.g., coadministration of drugs such as ursodeoxycholic acid and/or antibiotics, antibiotic-eluting stents, incorporating with hydrophilic coating technology, and adding of antireflux valve). ${ }^{10-17}$ However, promising outcomes have not derived until now. For instance, results between in vitro and in vivo studies were not consistent.

This study aimed to develop PS that may demonstrate longer patency and higher biodurability, by novel design with advanced hydrophilic coating technology.

\section{MATERIALS AND METHODS}

The current study is a preclinical, proof-of-concept study. This animal study was approved by the institutional animal care committee of Medi Kinetics Co., Ltd, contract research organization for preclinical test prior to the commencement of the study

Correspondence to: Seok Jeong

Department of Internal Medicine, Inha University Hospital, 27 Inhang-ro, Jung-gu, Incheon 22332, Korea

Tel: +82-32-890-2548, Fax: +82-32-890-2549, E-mail: inos@inha.ac.kr

Received on August 25, 2015. Revised on September 30, 2015. Accepted on September 30, 2015. Published online March 30 , 2016

pISSN 1976-2283 eISSN 2005-1212 http://dx.doi.org/10.5009/gnl15417

Chang-Il Kwon and Gwangil Kim contributed equally to this work as first authors.

@ This is an Open Access article distributed under the terms of the Creative Commons Attribution Non-Commercial License (http://creativecommons.org/licenses/by-nc/4.0) which permits unrestricted non-commercial use, distribution, and reproduction in any medium, provided the original work is properly cited. 
(MK-IACUC: 140103-001). Using an in vitro bile flow phantom model, we compared the patency between prototype PS with hydrophilic coating (PS+HC) and prototype PS without hydrophilic coating (PS-HC). Using an in vivo swine bile duct dilation model, we evaluated biodurability of hydrophilic coating. We compared four kinds of PS; polyethylene PS without hydrophilic coating (PE-HC), polyethylene PS with hydrophilic coating (PE+HC), polyurethane PS without hydrophilic coating (PU-HC), and polyurethane PS with hydrophilic coating (PU+HC).

\section{Prototype PS with hydrophilic coating}

Two types of commercially available 10-F plastic tubing (polyurethane tube of outer diameter $3.3 \mathrm{~mm}$ and inner diameter $2.0 \mathrm{~mm}$; polyethylene tubing of outer diameter $3.3 \mathrm{~mm}$ and inner diameter $2.0 \mathrm{~mm}$ ) were used as PS by cutting into 5 $\mathrm{cm}$ segments without any flap or side-hole. For the advanced hydrophilic coating on inside of PS, a bilaminar coating method was utilized with a base coating solution (Hydak B-23K; Biocoat Inc., Horsham, PA, USA) and a top coating solution (Hydak T-060; Biocoat Inc.). Bilaminar coating is commercially known to enhance coating durability and uniformity as compared to single laminar coating. Compared to single coating, which has coating coherence and performance deviations because the

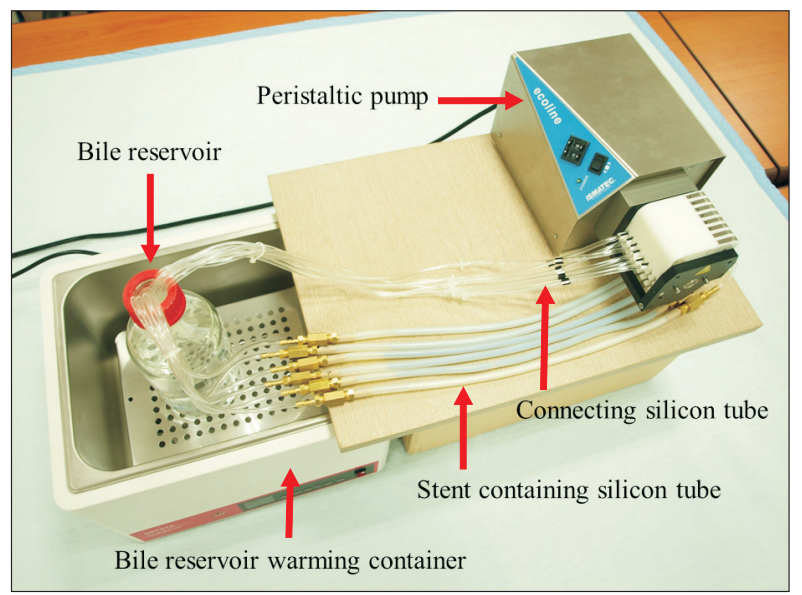

Fig. 1. An experimental perfusion system for the bile flow phantom model. This system consists of a bile reservoir, a peristaltic pump, a bile reservoir warming container, six connecting silicone tubes and six stent-containing silicone tubes. Six types of 10-F plastic tubes were inserted into the silicon tubes (36 stents in total).
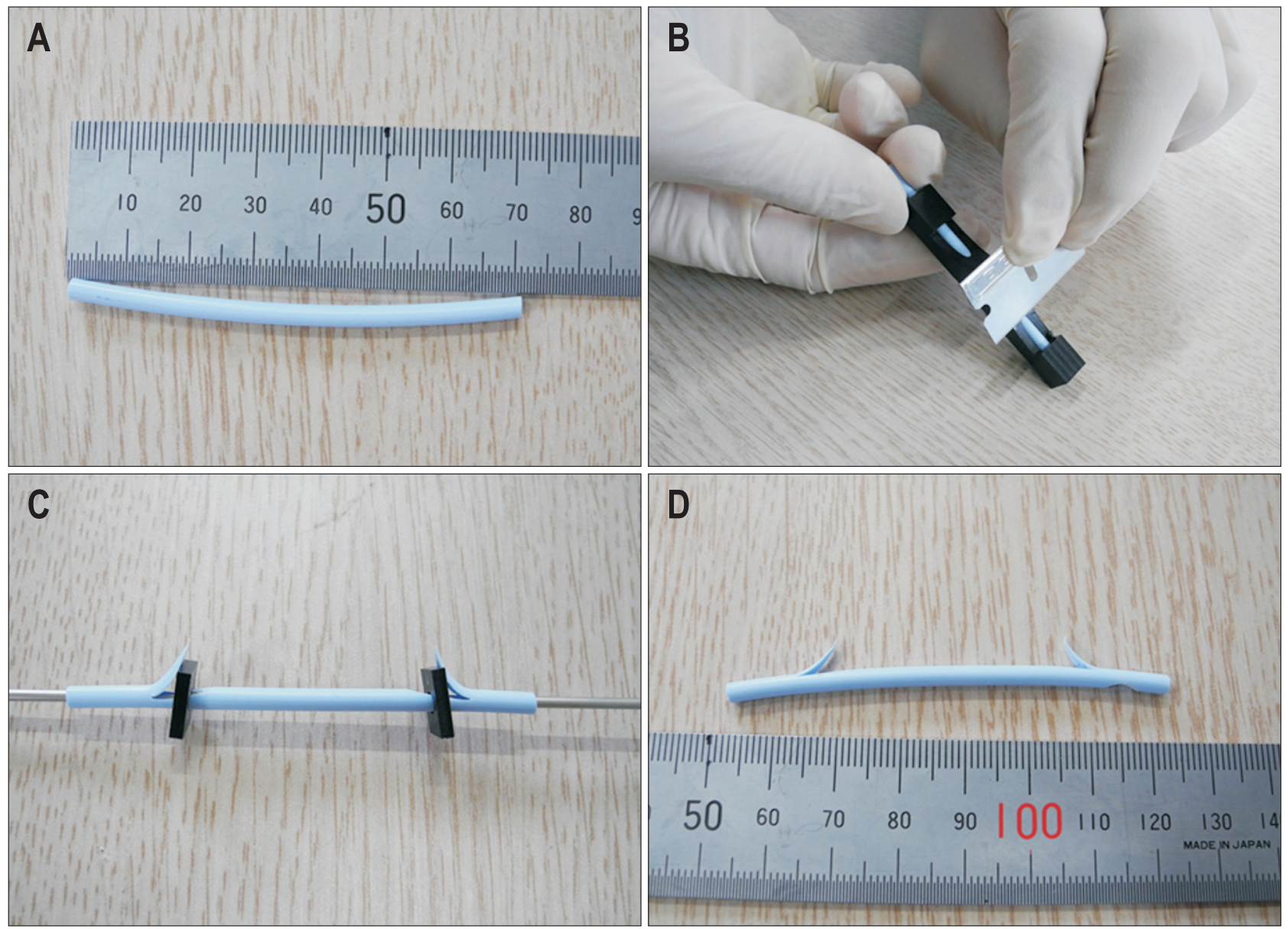

Fig. 2. The production of 10-F plastic tubes with bilateral flaps. (A) A commercially available plastic tube was cut to $7 \mathrm{~cm}$ (actual length of the 5 $\mathrm{cm}$ straight plastic stents). (B) The tube was fixed to a frame, and flaps were made on both sides using a blade. (C, D) The flaps were fixed to the frame so that they could maintain the shape, and a side hole was made at the proximal end. 
coating materials have to be combined directly with the PS, the bilaminar coating uses a highly coherent coating material for the base coating and a highly lubricative coating material for the top coating to make surface uniformity, regardless of the material or surface condition of the PS. The tubes were coated with the coating materials by using syringes with repeated dry process. As for the drying condition in a drying oven (Sungchan science, Pocheon, Korea), the optimal parameters (1 L/min for 20 minutes at $60^{\circ} \mathrm{C}$ ) were determined by attempting different dry temperatures and dry flow parameters.

\section{In vitro phantom model}

Using an in vitro bile flow phantom model, we compared patency between PS+HC and PS-HC. An experimental perfusion system for the bile flow phantom model was constructed following the previously reported method (Fig. 1). ${ }^{13,18}$ This system consists of a bile reservoir, a peristaltic pump, a bile reservoir warming container, six connecting silicone tubes, and six stentcontaining silicone tubes (six different types of stents were inserted serially into each silicone tube). Four PS groups were prepared; PE-HC, PE+HC, PU-HC, and PU+HC. Two types of commercially available 10-F PS from two different companies (A stent: Cotton-Leung ${ }^{\circledR}$ biliary stent, Cook Medical, Winston-
Salem, NC, USA; B stent: Percuflex ${ }^{\mathrm{TM}}$ biliary stent, Boston Scientific Corp., Natick, MA, USA) were used as control PS. These controls were made into the same shape as the test PS by cutting out both flanged-areas and leaving the 5-cm center area. The outer and inner diameters of control PS A were $3.3 \mathrm{~mm}$ and $2.0 \mathrm{~mm}$, respectively. The outer and inner diameters of control PS B were $3.4 \mathrm{~mm}$ and $2.5 \mathrm{~mm}$, respectively. A total of $600 \mathrm{~mL}$ of human bile obtained by drainage catheter in patients with cholangitis was replaced every 2 days in the bile flow phantom. The peristaltic pump was set so that the bile could recirculate at $1 \mathrm{~mL} / \mathrm{min}$. At 4 weeks and 8 weeks after bile exposure, the stentcontaining silicone tubes were removed to examine the stents.

\section{In vivo animal model}

For the in vivo studies, the PS were cut into $7 \mathrm{~cm}$ (actual length of the $5 \mathrm{~cm}$ straight PSs) and flaps were made to prevent migration at both ends. A side hole was made at the proximal portion of the stents, making the stents look almost similar to a 5-cm, 10-F conventional stents (Fig. 2).

\section{1) Animals}

Seven mini-pigs (Sus scrofa, mean age 14 months, mean body weight $29.9 \mathrm{~kg}$ ) were used for current study. The pigs were
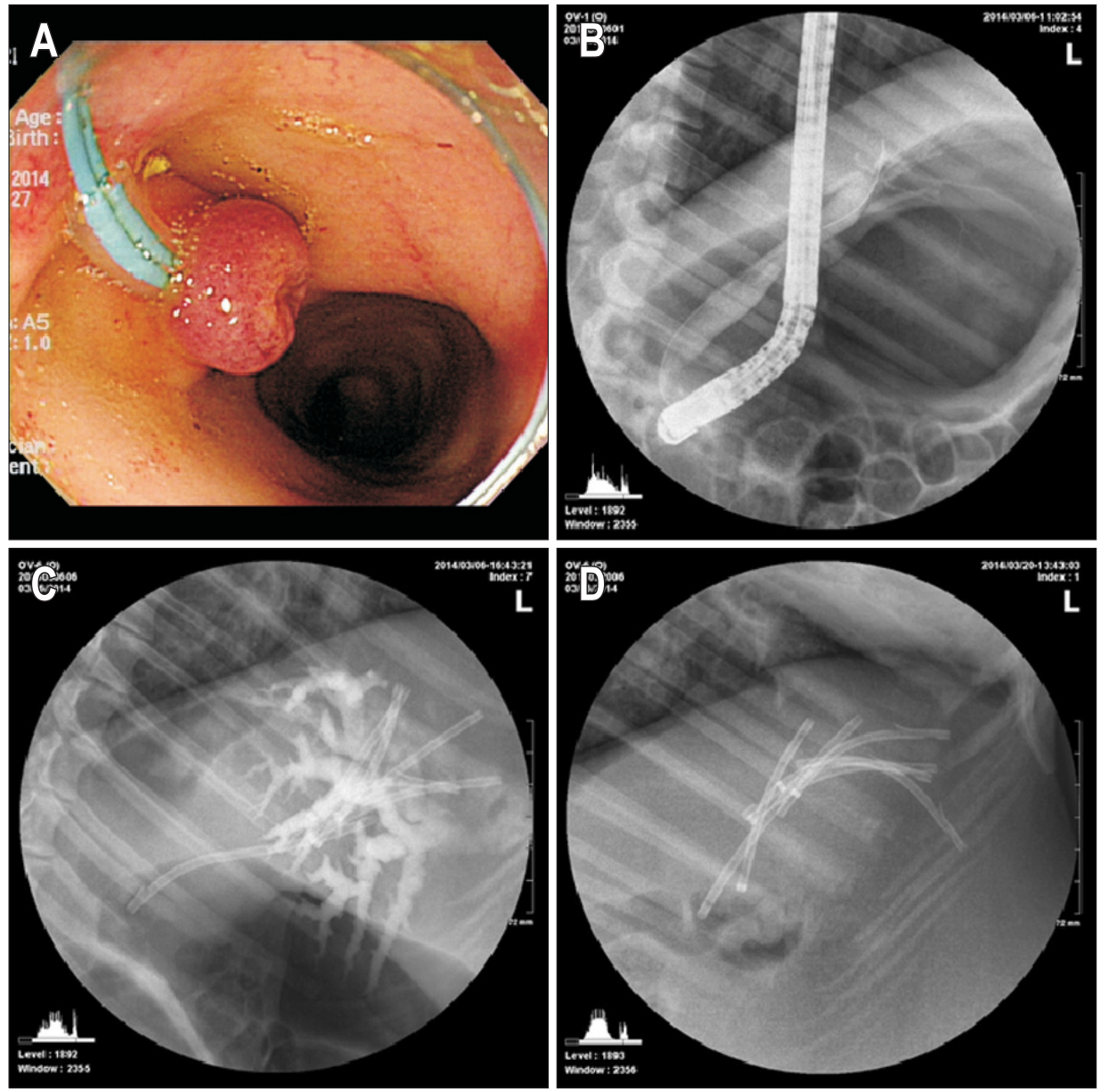

Fig. 3. Endoscopic procedures in the animal bile duct dilation model. (A) A detachable snare obstructed the major duodenal papilla. (B) Representative cholangiography showing marked biliary dilation 2 weeks after endoscopic papillary closure. (C) Six plastic stents were successfully inserted into the dilated biliary duct on a representative specimen. (D) Follow-up fluoroscopic examination of the same specimen reveals plastic stents that remain in the biliary tract. 
fasted overnight, except for water that was given ad libitum, before the endoscopic procedure. Preanesthesia sedation consisted of an intramuscular injection of atropine sulfate $(0.04 \mathrm{mg} / \mathrm{kg})$, xylazine (2 mg/kg), and tiletamine-zolazepam (5 mg/kg). Animals were intubated and general anesthesia was achieved using $0.5 \%$ to $2 \%$ isoflurane through an endotracheal tube with $70 \%$ nitrous oxide and 30\% oxygen provided by a ventilator, and then placed on their sides on a fluoroscopy table.

\section{2) Endoscopic procedures}

Endoscopic papillary closure was performed with a transparent cap-fitted conventional endoscope (GIF-Q260J; Olympus Co., Tokyo, Japan) using a detachable snare (MAJ-340; Olympus Co.), according to previously reported our method (Fig. 3A). ${ }^{19}$ After positioning the endoscope close to the major papilla, a detachable snare was positioned around the base of the papilla and tightened, and then the stopper was detached from the device.

\section{3) Endoscopic retrograde cholangiography and biliary stents insertion}

Two weeks after the endoscopic papillary closure, endoscopic retrograde cholangiography (ERC) using a side-viewing duodenoscope (TJF-240; Olympus Co.) was implemented to confirm biliary tree dilation and to insert the PS (Fig. 3B-D). After duodenal intubation of the scope, biliary cannulation (ERCP catheter, bottle-shaped metal tip; MTW Endoskopie, Wesel, Germany) was performed using the wire-guided cannulation method with a 0.035-inch hydrophilic tipped guidewire (Boston Scientific Corp.). After obtaining cholangiogram, two types of PS+HC and two types of conventional PS were inserted serially into each bile duct of seven swine bile duct dilation models. According to the degree of bile duct dilation, maximum number of PS insertion was determined in each swine as many as possible. Fluoroscopic images were repeated every 2 weeks after the procedures for the identification of remaining PS in the biliary tract. After fluorography at 8 weeks, all live swine were euthanized and all
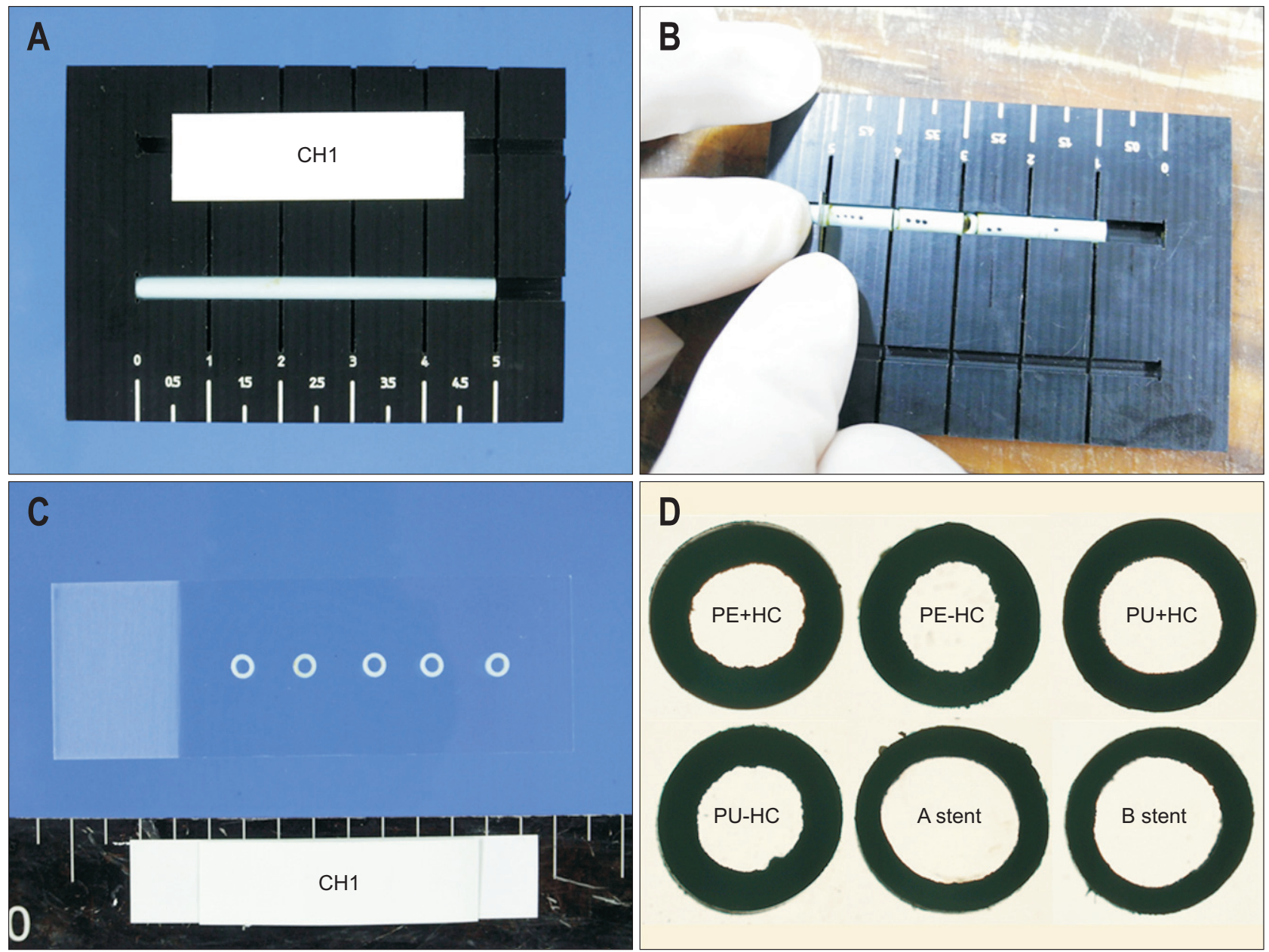

Fig. 4. Light microscopic patency evaluation for the retrieved stents. (A) The retrieved 5-cm plastic stents were fixed to a self-produced frame. (B) The stents were cut into sections at 10-mm intervals using a blade, and then, a 1-mm small section was made again at one end of the 10-mm sections. (C) Five small sections made from one stent were placed on a slide, and their luminal patency was observed microscopically. (D) Representative samples of the lumens were observed at 8 weeks with a microscope $(\times 12)$.

$\mathrm{PE}$, polyethylene; PU, polyurethane; HC, hydrophilic coating. 
remaining PS in the bile ducts were removed.

\section{Light microscopic evaluation}

Gross inspection and light microscopic (LM) examination were performed by a pathologist (G.K.) who was blinded to the types of PS. The retrieved 5-cm PSs were fixed to a custommade frame and cut each section into 10-mm tubes using R35 Feather disposable microtome blades (Feather Safety Razor Co., Osaka, Japan). A small section of 1-mm length was made again at one end of each of these $10-\mathrm{mm}$ sections. LM examination was performed to observe luminal patency of these small sections (Fig. 4). The exact quantitative measurements of the patency of retrieved stents were performed using Image $1.47 \mathrm{~V}$ (National Institute of Health, Bethesda, MD, USA). The percent of remaining cross-section area of the inner lumen was calculated by dividing the area of inner patent lumen at 4 weeks and 8 weeks by the area of original inner lumen of each stent.

\section{Scanning electron microscopy examination}

Apart from the above mentioned 1-mm section for LM examination, the remaining 4-mm sections were cut vertically to perform scanning electron microscopy (SEM) (S-4800; Hitachi, Tokyo, Japan) on the inner surface. After coating the surface with $200 \mathrm{~nm}$ of platinum, constant magnification was performed to evaluate hydrophilic coating durability and biodurability.

\section{Statistical analysis}

One-way ANOVA test and the Student t-test were used to analyze the difference of $\%$ stent patency area. A p-value $<0.05$ was considered significant. Statistical analysis was performed with IBM SPSS version 21.0 (IBM Corp., Armonk, NY, USA).

\section{RESULTS}

\section{In vitro assessment of stent patency area in six types of PS}

Four weeks after the stent insertion, three silicon tubes harboring PS were removed from the in vitro bile flow phantom and the 18 PS were retrieved. Biofilm was not visibly formed via LM examination in any of the 18 stents regardless of their

Table 1. Detailed Statistical Analysis of Stent Patency at 8 Weeks after Stent Insertion in the Bile Phantom Model

\begin{tabular}{cccccc}
\hline \multirow{2}{*}{ Group } & \multicolumn{5}{c}{ p-value } \\
\cline { 2 - 6 } & PE+HC & PE-HC & PU+HC & PU-HC & A stent \\
\hline PE-HC & 0.131 & - & - & - & - \\
PU+HC & 0.322 & 0.017 & - & - & - \\
PU-HC & 0.004 & 0.048 & $<0.001$ & - & - \\
A stent & 0.42 & 0.065 & 0.794 & 0.002 & - \\
B stent & 0.063 & 0.006 & 0.158 & $<0.001$ & 0.419 \\
\hline
\end{tabular}

Student t-test.

PE, polyethylene; PU, polyurethane; HC, hydrophilic coating. types at 4 weeks (data not shown). The remaining three silicon tubes were removed after 8 weeks and when these stents were examined under LM (Fig. 4D), PS without hydrophilic coating (PS-HC) showed significantly decreased stent patency compared to PS+HC and conventional stents $(\mathrm{p}<0.05)$ (Table 1, Fig. 5). The percent patency areas according to stent types are as follows: PE-HC, 92.9 \pm 2.7 ; PE+HC, 94.3 \pm 2.0 ; PU-HC, 90.2 \pm 6.4 ; PU+HC,

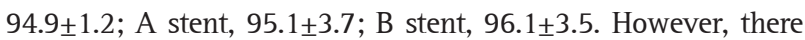
was no significant difference between hydrophilic coating groups and conventional stent groups.

\section{In vivo assessment of stent patency area according to four types of PS}

Two weeks after papillary obstruction, the porcine bile duct diameter was dilated to a mean of $16.4 \pm 3.2 \mathrm{~mm}$. A total of 31 stents (8 PE+HC, 8 PU+HC, 7 A stents, and 8 B stents) were inserted into the bile ducts of the seven swine models, ranging from minimum 3 to maximum 6 stents in each swine bile duct. Although most stents were inserted with the above the papilla method for preventing stent migrations, some final stents were necessarily inserted with the across the papilla method. The swine fared well without any remarkable event throughout 8 weeks. The number of inserted stents was followed by fluoroscopic images every 2 weeks. Seven out of the 31 inserted stents were lost during the observation period (stents loss rate, 22.6\%), and 24 stents (6 PE+HC, 7 PU+HC, 5 A stents, and 6 B stents) were retrieved from the dilated bile ducts at 8 weeks after the insertion.

On the LM examination of the retrieved stents, biofilms were definitely formed and the decrease in patency was much more prominent compared to the in vitro phantom (Fig. 6). The difference among the groups was not statistically significant ( $p=0.407$ ). Also, there was no statistical difference of stent patency between the $\mathrm{PE}+\mathrm{HC}$ and the $\mathrm{PU}+\mathrm{HC}(\mathrm{p}=0.062)$ (Fig. 7). The percent

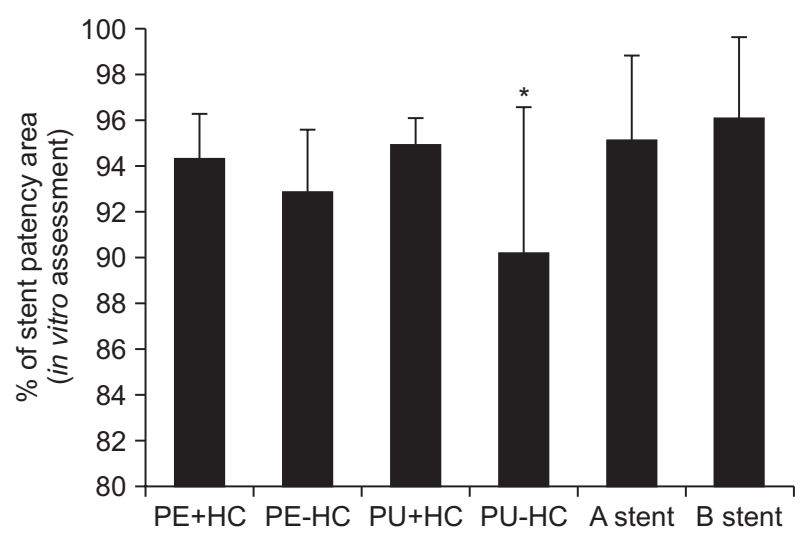

Fig. 5. Result of stent patency at 8 weeks after stent insertion in the bile phantom model. The $\%$ of stent patency area was measured low in the stent groups without hydrophilic coating (one-way analysis of variance, ${ }^{*} \mathrm{p}<0.001$ ). $\mathrm{PE}$, polyethylene; PU, polyurethane; HC, hydrophilic coating. 


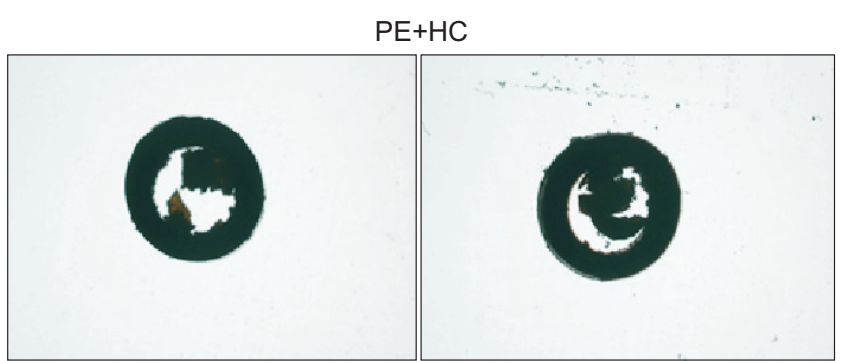

A stent

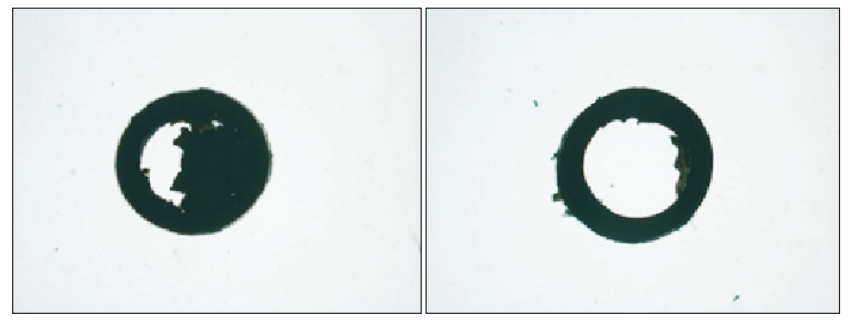

Fig. 6. Examples of light microscopic examination for retrieved stents in the animal bile duct dilation model. Biofilm formation was observed and the patency area was reduced in the sections $(\times 12)$.

PE, polyethylene; PU, polyurethane; HC, hydrophilic coating.

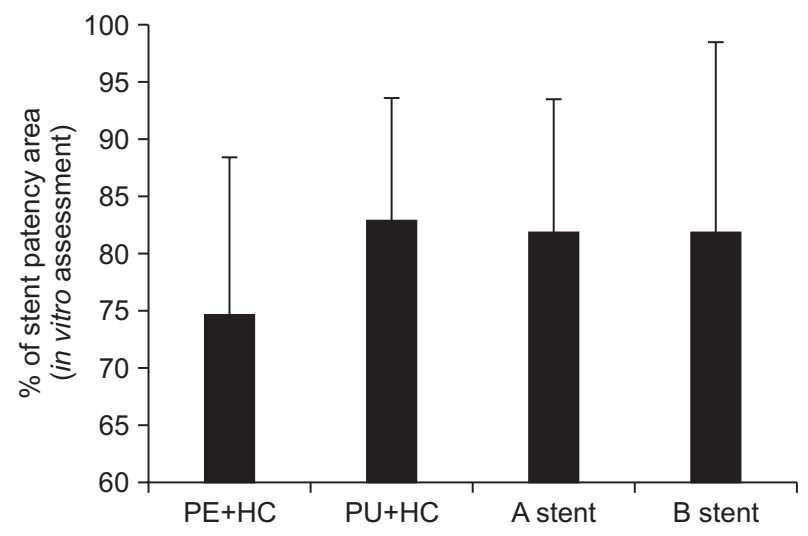

Fig. 7. Result of stent patency at 8 weeks after stent insertion in the animal bile duct dilation model. The PE+HC had a tendency to form more biofilm and constrict the lumen than the other types of stents (one way analysis of variance, $\mathrm{p}=0.407$ ).

$\mathrm{PE}$, polyethylene; PU, polyurethane; HC, hydrophilic coating.

patency areas were as follows: $\mathrm{PE}+\mathrm{HC}, 74.6 \pm 11.4$; $\mathrm{PU}+\mathrm{HC}$,

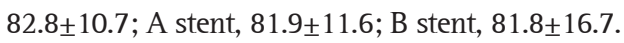

\section{SEM examination}

SEM examination was performed to observe the hydrophilic coating layer in all sections of the stents retrieved from the two models. On the SEM analysis of the stents at 4 weeks of in vitro bile exposure, biofilm formation was almost nonexistent and the top coating layer was virtually invisible, but most of the base coating layer was still in place (Fig. 8). On the analysis of the stents at 8 weeks of in vitro bile exposure, biofilm formation was almost formed in all types of stents and most of the base coating layer still existed (Fig. 9A and B). In the swine model, the biofilm layer was generally thicker and the base coating layer was relatively thinner (Fig. 9C and D).

\section{DISCUSSION}

To overcome stent occlusion in biliary PS, SEMS with a larger diameter or new SEMS with additional function have been or are currently being developed. However, as SEMS is hard to insert and remove, and more of a burden in price, many researches on PS are also continuously ongoing these days. For instance, to improve the function and patency of PS, inserting multiple PS, and changing the internal diameter or shape of PS have been tried. ${ }^{17,20-23}$ In spite of these efforts, these attempts are not supported by additional clinical researches or follow-up studies confirming the effectiveness.

Based on the fact that bacterial adhesion to the plastic surface is closely related to the surface hydrophobicity, ${ }^{14,24}$ multiple studies regarding hydrophilic coating onto PS have been started. ${ }^{15,25}$ However, further advancements are still prerequisite because they just covered short-term changes and they could not quantify total amount of biofilm formation itself. Two prospective randomized controlled studies were performed based on the results of in vitro studies that hydrophilic coating had positive effects on stent patency. A randomized controlled trial by Costamagna et al. ${ }^{16}$ showed a tendency toward longer patency in PU+HC group, but the difference was not statistically significant. The other clinical study is a prospective randomized trial by van Berkel et al. ${ }^{12}$ showed contradictory result that PE stent yielded higher stent patency than PU+HC stent.

There are two possible explanations for the conflicting results. The first one is the same as the speculation in the previous study; manipulation at the time of stent placement and coating surface damage from duodenal reflux, although the SEM 

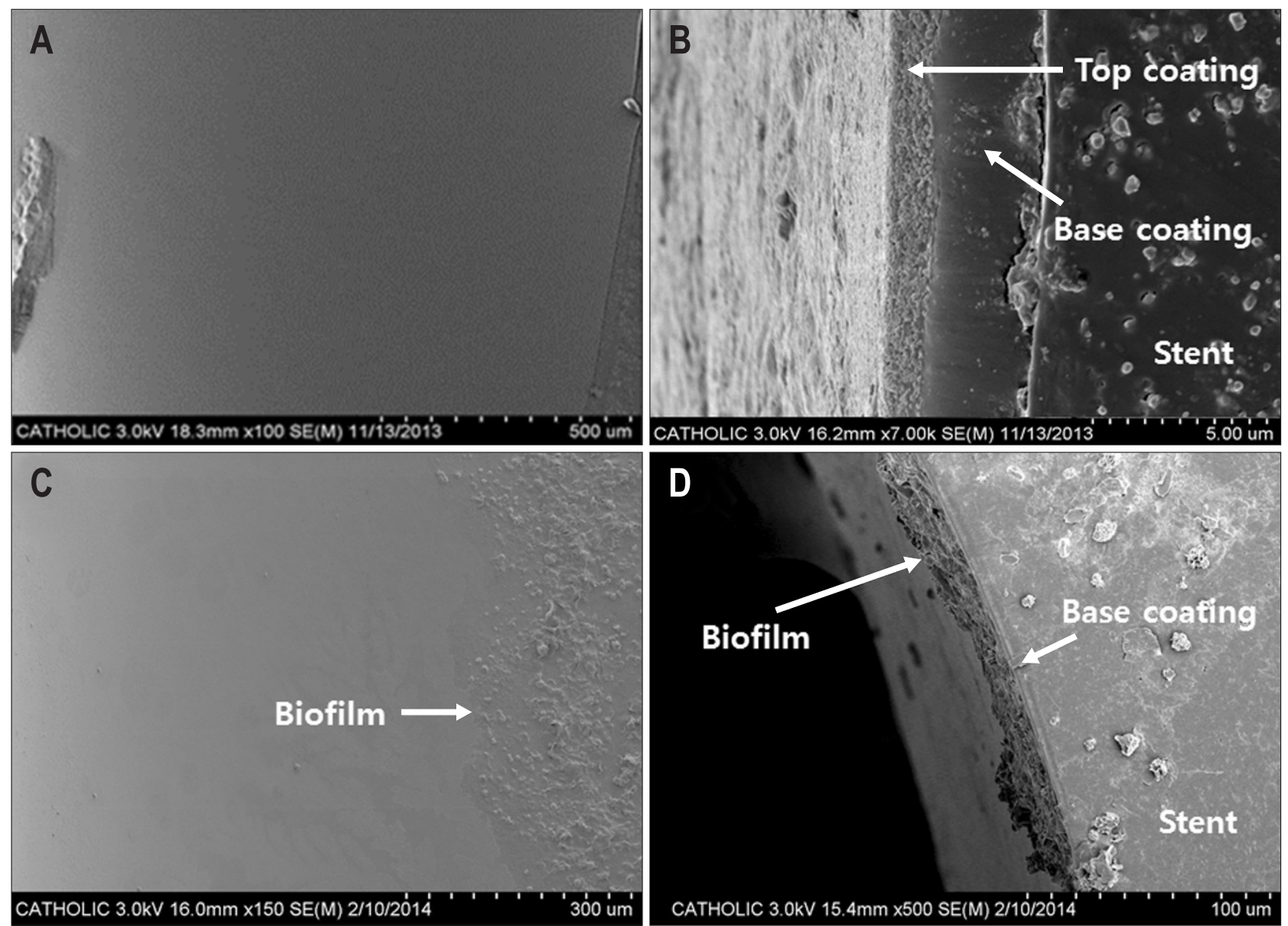

Fig. 8. Scanning electron microscopy images of the polyethylene plastic stents with a hydrophilic coating. After bilaminar hydrophilic coating and drying processes, the bilaminar coating was performed relatively well $(\mathrm{A}, \times 100 ; \mathrm{B}, \times 7,000)$. At 4 weeks of in vitro bile exposure, biofilm formation was almost nonexistent and the top coating layer was virtually invisible, but most of the base coating layer was still in place (C, $\times 150 ; \mathrm{D}, \times 500)$.

examination after manipulation did not support this idea. The second one is that the coating layer would have been degraded with time, which was a mere speculation.

Stimulated with these contradictory results, we conducted this study. According to our results of the in vitro phantom model, biofilm formation by human bile was almost nonexistent in the stents retrieved at 4 weeks, which was why it was impossible to measure inner diameter change. It has been known that a stent design without side holes reduces micro-turbulence of bile flow, producing less biofilm formation in theory, although the theory has not been supported by clinical evidences. ${ }^{5,26-28}$ Lowered formation of biofilm at 4 weeks might be possibly explained by the removal of both flanges areas before inserting the stents. In the stents retrieved at 8 weeks, the PS+HC showed less biofilm formation and less luminal narrowing than PS-HC $(\mathrm{p}<0.05)$. Given that biofilm in the phantom model at 8 weeks was not as apparent as in vivo model, it would have been useful to extend the trial beyond 8 weeks. But this period was selected because it is generally deemed safe for PSs before total obstruction. If a total occlusion occurs in one stent, serial occlusions can quickly occur in other stents in a same harboring silicon tube. At any rate, measurements at 4 and 8 weeks seem to have yielded interesting results for the purpose of evaluating the biodurability of the hydrophilic coating, which will be described again in the SEM analysis.

The biggest concern in the in vivo animal model was that we could not be sure of the maximum number of PSs that can be inserted after biliary dilation and whether the stents could be maintained for 8 weeks, unlike the expectation that biliary duct dilation model would be made easily following the method we have previously reported. ${ }^{19}$ In the current study, three to six PSs were inserted in each swine bile duct and a total of 31 stents were inserted with both flanges intact unlike the in vitro phantom model. Seven out of the 31 inserted stents were lost, with a relatively good stent loss rate (22.6\%). Under more severe conditions, considering the possibility of additional effects, such as duodenal reflux, unlike the in vitro phantom model, mean stent patency area was decreased in every type of stent. When SEM analysis was used to determine whether the severe conditions also affected the hydrophilic coating, the biofilm layer was 

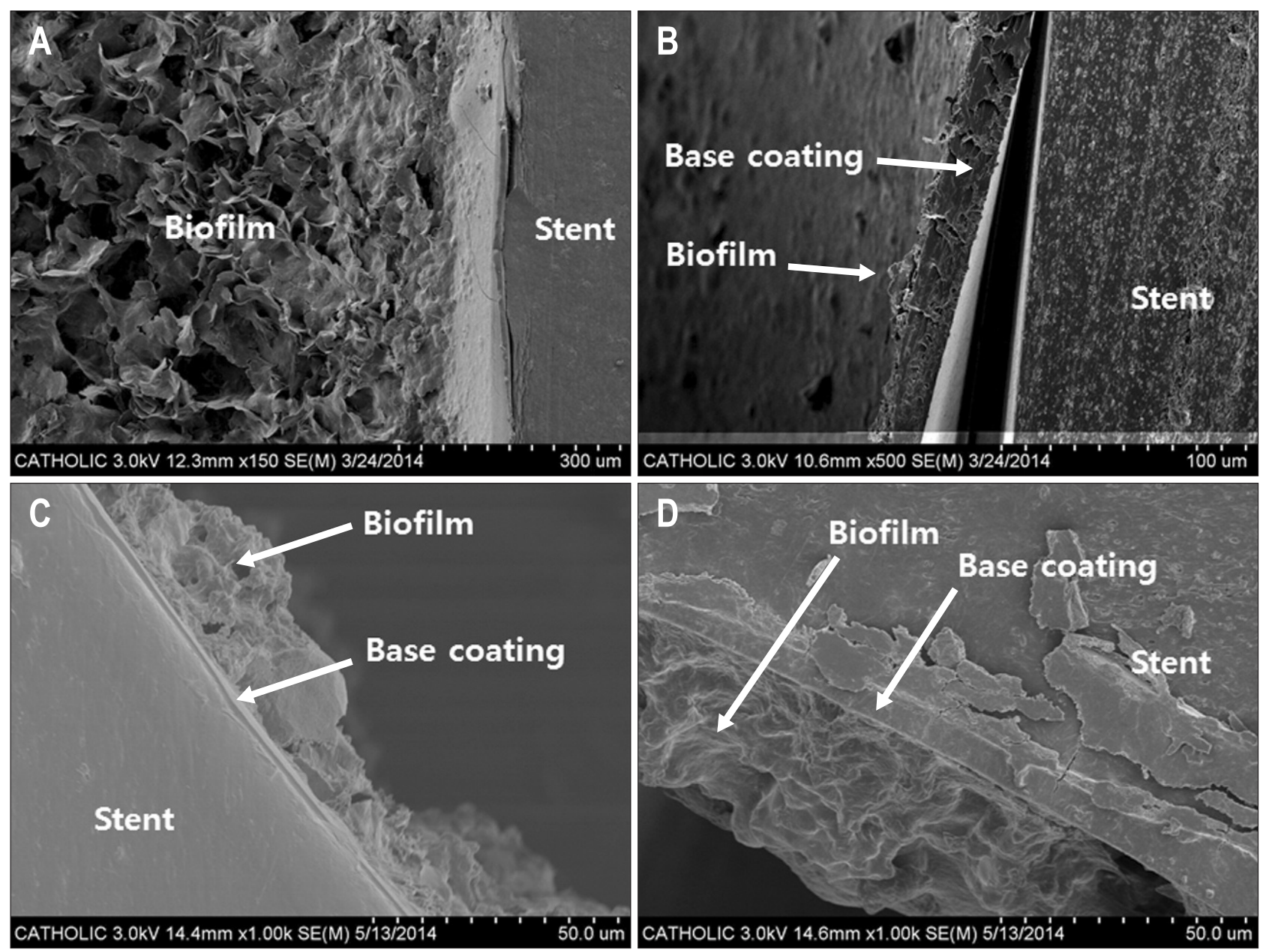

Fig. 9. Scanning electron microscopy images representing each stent type retrieved at 8 weeks. By 8 weeks, the top coating layer was invisible with biofilm formation, but the base coating layer was still present. (A) PE+HC in the in vitro phantom model $(\times 150)$, (B) PU+HC in the in vitro phantom model $(\times 500),(\mathrm{C}) \mathrm{PE}+\mathrm{HC}$ in the in vivo animal model $(\times 1,000)$, (D) PU+HC in the in vivo animal model $(\times 1,000)$.

$\mathrm{PE}$, polyethylene; PU, polyurethane; HC, hydrophilic coating.

generally thicker and the base coating layer was thinner in the in vivo model, as can be seen in Fig. 9, although statistical difference was not obtained. SEM images in the in vitro model at 4 weeks showed the stents began losing the top coating layer, suggesting that biofilm formation might have started afterward, only inhibited by the remaining base coating layer. We could infer a correlation of biofilm formation is negatively associated with the hydrophilic coating presence and the base coating presence.

This report is likely the first proof-of-concept study using an endoscopic biliary dilation porcine model with realistic ERC stent insertion. The fact that the durability of stents could be prolonged to 8 weeks by using our model, not a surgical model, is academically of great significance. This mode of investigation into experimental biliary devices may hold great promise for future studies. Another significance of the current study is that we demonstrated with the bilaminar coating enhanced the biodurability of the stents, inhibiting biofilm formation and thus improving stent patency, unlike previous reports which suggest- ed that hydrophilic coating layer lasts only for a short period of time and may not provide a long-term inhibition of biofilm formation. Although our study could not observe the superiority of the hydrophilic coating stent than other conventional stents, this study looked into the technical features of the hydrophilic coating stent with bilaminar coating method, and is deemed meaningful as a noninferiority test.

Our study has the following limitations; being an in vitro and in vivo animal model, not an in vivo human study, the difference of bile acid composition and hydrophilicity (hydrophobichydrophilic balance) between human and swine, and the inner diameter of the prototype stents may not be identical to the inner diameter of commercially available stents. Nevertheless, we could conclude that advanced hydrophilic coating technology led to less forming of biofilm in PS than uncoated PS. Further studies are needed to demonstrate the beneficial effect of hydrophilic coating on biofilm formation more accurately and in a more quantitative way. Thereafter, human studies would be essential to support superior effects of advanced hydrophilic coat- 
ing technology prior to general acceptance. Also, in addition to bile flow phantom model, swine bile duct dilation model made by endoscopic procedure can be useful for PS evaluation.

\section{CONFLICTS OF INTEREST}

No potential conflict of interest relevant to this article was reported.

\section{ACKNOWLEDGEMENTS}

The following author received research support for this study from the Industrial Technology Innovation Program (Advanced Technology Center Program) funded by the Korean Ministry of Trade, Industry \& Energy and the National Center of Efficacy Evaluation for the Development of Health Products Targeting Digestive Disorders (NCEED): Chang-Il Kwon.

We would like to thank Mark A. Gromski, M.D. for his critical review of the manuscript prior to publication, and thank Yong Hyun Won, Kil Soo Kim, and Se Il Kang (Institute of Technology, Taewoong Medical Co., Ltd., Goyang, Korea) for their devoted cooperation during study.

\section{REFERENCES}

1. Raijman I. Biliary and pancreatic stents. Gastrointest Endosc Clin N Am 2003;13:561-592.

2. Kwon CI, Ko KH, Hahm KB, Kang DH. Functional self-expandable metal stents in biliary obstruction. Clin Endosc 2013;46:515-521.

3. Kadakia SC, Starnes E. Comparison of 10 French gauge stent with 11.5 French gauge stent in patients with biliary tract diseases. Gastrointest Endosc 1992;38:454-459.

4. van Berkel AM, Boland C, Redekop WK, et al. A prospective randomized trial of Teflon versus polyethylene stents for distal malignant biliary obstruction. Endoscopy 1998;30:681-686.

5. England RE, Martin DF, Morris J, et al. A prospective randomised multicentre trial comparing 10 Fr Teflon Tannenbaum stents with 10 Fr polyethylene Cotton-Leung stents in patients with malignant common duct strictures. Gut 2000;46:395-400.

6. Terruzzi V, Comin U, De Grazia F, et al. Prospective randomized trial comparing Tannenbaum Teflon and standard polyethylene stents in distal malignant biliary stenosis. Gastrointest Endosc 2000;51:23-27.

7. Guaglianone E, Cardines R, Vuotto C, et al. Microbial biofilms associated with biliary stent clogging. FEMS Immunol Med Microbiol 2010;59:410-420.

8. Leung JW, Liu Y, Chan RC, et al. Early attachment of anaerobic bacteria may play an important role in biliary stent blockage. Gastrointest Endosc 2000;52:725-729.

9. van Berkel AM, van Marle J, Groen AK, Bruno MJ. Mechanisms of biliary stent clogging: confocal laser scanning and scanning electron microscopy. Endoscopy 2005;37:729-734.
10. Galandi D, Schwarzer G, Bassler D, Allgaier HP. Ursodeoxycholic acid and/or antibiotics for prevention of biliary stent occlusion. Cochrane Database Syst Rev 2002;(3):CD003043.

11. Gwon DI, Lee SS, Kim EY. Cefotaxime-eluting covered self-expandable stents in a canine biliary model: scanning electron microscopic study of biofilm formation. Acta Radiol 2012;53:11271132.

12. van Berkel AM, Bruno MJ, Bergman JJ, van Deventer SJ, Tytgat GN, Huibregtse K. A prospective randomized study of hydrophilic polymer-coated polyurethane versus polyethylene stents in distal malignant biliary obstruction. Endoscopy 2003;35:478-482.

13. Hoffman BJ, Cunningham JT, Marsh WH, O’Brien JJ, Watson J. An in vitro comparison of biofilm formation on various biliary stent materials. Gastrointest Endosc 1994;40:581-583.

14. Sung JY, Shaffer EA, Lam K, Rususka I, Costerton JW. Hydrophobic bile salt inhibits bacterial adhesion on biliary stent material. Dig Dis Sci 1994;39:999-1006.

15. Jansen B, Goodman LP, Ruiten D. Bacterial adherence to hydrophilic polymer-coated polyurethane stents. Gastrointest Endosc 1993;39:670-673.

16. Costamagna G, Mutignani M, Rotondano G, et al. Hydrophilic hydromer-coated polyurethane stents versus uncoated stents in malignant biliary obstruction: a randomized trial. Gastrointest Endosc 2000;51:8-11.

17. Dua KS, Reddy ND, Rao VG, Banerjee R, Medda B, Lang I. Impact of reducing duodenobiliary reflux on biliary stent patency: an in vitro evaluation and a prospective randomized clinical trial that used a biliary stent with an antireflux valve. Gastrointest Endosc 2007;65:819-828.

18. Bang BW, Jeong S, Lee DH, Lee JI, Lee SC, Kang SG. The biodurability of covering materials for metallic stents in a bile flow phantom. Dig Dis Sci 2012;57:1056-1063.

19. Park JS, Kwon CI, Jeong S, Kim K, Moon JH, Lee DH. Development of a swine bile duct dilation model using endoclips or a detachable snare under cap-assisted endoscopy. Gastrointest Endosc 2014;80:325-329.

20. Elwir S, Sharzehi K, Veith J, et al. Biliary stenting in patients with malignant biliary obstruction: comparison of double layer, plastic and metal stents. Dig Dis Sci 2013;58:2088-2092.

21. Guo Y, Liu Y, Lu Z, et al. Obstructive component analysis of radioactive stents and common plastic stents in the bile duct. Eur J Gastroenterol Hepatol 2014;26:795-802.

22. Cai XB, Zhang WX, Wan XJ, et al. The effect of a novel drugeluting plastic stent on biliary stone dissolution in an ex vivo bile perfusion model. Gastrointest Endosc 2014;79:156-162.

23. Farnbacher MJ, Lederer R, Blana A, Schneider HT. Does heparin coating reduce encrustation of biliary plastic endoprostheses? A prospective randomized trial. Scand J Gastroenterol 2012;47:11411147.

24. John SF, Hillier VF, Handley PS, Derrick MR. Adhesion of staphylococci to polyurethane and hydrogel-coated polyurethane catheters assayed by an improved radiolabelling technique. J Med 
Microbiol 1995;43:133-140.

25. McAllister EW, Carey LC, Brady PG, Heller R, Kovacs SG. The role of polymeric surface smoothness of biliary stents in bacterial adherence, biofilm deposition, and stent occlusion. Gastrointest Endosc 1993;39:422-425.

26. Seitz U, Vadeyar H, Soehendra N. Prolonged patency with a newdesign Teflon biliary prosthesis. Endoscopy 1994;26:478-482.
27. Sung JJ, Chung SC, Tsui CP, Co AL, Li AK. Omitting side-holes in biliary stents does not improve drainage of the obstructed biliary system: a prospective randomized trial. Gastrointest Endosc 1994;40:321-325.

28. Binmoeller KF, Seitz U, Seifert H, Thonke F, Sikka S, Soehendra N. The Tannenbaum stent: a new plastic biliary stent without side holes. Am J Gastroenterol 1995;90:1764-1768. 